\title{
Commentary \\ Advances in breast cancer treatment and prevention: preclinical studies on aromatase inhibitors and new selective estrogen receptor modulators (SERMs)
}

\author{
Rachel Schiff, Gary C Chamness and Powel H Brown \\ The Breast Center, Department of Medicine, Baylor College of Medicine, Houston, TX, USA \\ Corresponding author: Rachel Schiff (e-mail: rschiff@breastcenter.tmc.edu) \\ Published: 28 July 2003 \\ Breast Cancer Res 2003, 5:228-231 (DOI 10.1186/bcr626) \\ (c) 2003 BioMed Central Ltd (Print ISSN 1465-5411; Online ISSN 1465-542X)
}

\begin{abstract}
Intensive basic and clinical research over the past 20 years has yielded crucial molecular understanding into how estrogen and the estrogen receptor act to regulate breast cancer and has led to the development of more effective, less toxic, and safer hormonal therapy agents for breast cancer management and prevention. Selective potent aromatase inhibitors are now challenging the hitherto gold standard of hormonal therapy, the selective estrogen-receptor modulator tamoxifen. Furthermore, new selective estrogen-receptor modulators such as arzoxifene, currently under clinical development, offer the possibility of selecting one with a more ideal pharmacological profile for treatment and prevention of breast cancer. Two recent studies in preclinical model systems that evaluate mechanisms of action of these new drugs and suggestions about their optimal clinical use are discussed.
\end{abstract}

Keywords: aromatase inhibitors, arzoxifene, breast cancer, prevention, rexinoid

\section{Introduction}

Hormonal (endocrine) therapy $(\mathrm{HT})$ is one of the most effective treatments for breast cancer in the adjuvant, the metastatic, and the prevention settings. For more than two decades, the anti-estrogen tamoxifen has been the HT of choice for all stages of ER-positive breast cancer [1], and tamoxifen is still the only approved agent, in the United States, to reduce the risk of breast cancer in high-risk women. Tamoxifen is a prototype of a class of drugs called selective estrogen-receptor (ER) modulators (SERMs), which exhibit anti-estrogen effects in the breast but possess estrogen-like activity in other tissues such as bone and blood [1]. This inherent mixed agonist/antagonist nature of tamoxifen is probably responsible for the two major limits of its successful therapeutic promise, i.e. tumor resistance, de novo or acquired, seen in many patients, and its adverse effects in other tissues. Developments over the past two decades have led to potentially more effective, less toxic, and safer HT agents that are currently being implemented into the management of breast cancer, or soon will be. This, in turn, brings the challenge of determining the optimal use of these new drugs, either in combination or in sequence, questions that are currently under investigation in key preclinical models and clinical trials. Two recently reported preclinical studies - one by Long and colleagues [2] that demonstrates the efficacy of anti-estrogens as second-line therapy in breast tumors failing aromatase inhibitor (Al) therapy, and one by Suh and colleagues [3] that shows high synergism between arzoxifene and the new rexinoid LG 100268 in treatment and prevention - make significant contributions in this area and are discussed.

\section{Aromatase inhibitors: clinical efficacy}

Estrogen deprivation was suggested long ago as one of the most efficient strategies to block ER action [4]. After menopause, estrogen deprivation is most specifically achieved using inhibitors that block the conversion of adrenal androgens to estrogens by the enzyme aromatase [5]. The third-generation nonsteroidal Als anastrozole and 
letrozole have both shown, in postmenopausal women, superior efficacy compared with tamoxifen as first-line treatments for advanced breast cancer, and, at least for letrozole, also as neoadjuvant therapy for ER-positive invasive breast cancer (reviewed in [6]). Current results from the ATAC study in postmenopausal women with early ER-positive breast cancer further suggest that in the adjuvant setting, an Al (anastrozole) is superior to tamoxifen in terms of disease-free survival and in preventing contralateral incidents [7]. These Als may very soon occupy a central role in the management of postmenopausal women with hormone-dependent breast cancer. Previously, Als have proven effective in postmenopausal women with metastatic breast cancer in whom tamoxifen has failed [8]. Breast cancers in patients treated with Als as first-line therapy for metastatic disease are likely to eventually become resistant, but may still respond to another type of $\mathrm{HT}$. Therefore, as these Al agents move into the first line, it is essential to establish appropriate second-line therapies. This important clinical question was directly addressed by Long and colleagues [2], using a preclinical model of xenografts with intratumoral aromatase.

\section{Intratumoral aromatase preclinical model}

Clinical evidence suggesting that local production of estrogen may contribute to breast tumor growth and, therefore, that intratumoral aromatase is a potential therapeutic target [9] had led Brodie's research group, almost 10 years ago, to develop a valuable preclinical mouse model of intratumoral aromatase $[5,10]$. Tumors formed by ER-positive MCF-7 human breast cancer cells stably transfected with the human aromatase gene (MCF-7Ca) were grown in ovariectomized nude mice. These tumor cells remain hormone-dependent and, in the presence of aromatase substrate, synthesize sufficient estrogen to stimulate tumor formation and progression [5]. This system, which models the low-estrogen state in postmenopausal women, has proven to successfully predict some HT effectiveness in such women with breast cancer. Thus, previous studies from this model have shown that Als, and especially letrozole, are more effective at suppressing tumor growth than either tamoxifen or the pure potent anti-estrogen fulvestrant (ICl 182,780; Faslodex) [5,11], and that the combination of Als with anti-estrogens is no better than treatment with an $\mathrm{Al}$ alone. The superiority of $\mathrm{Al}$ agents over tamoxifen for the treatment of postmenopausal women with advanced breast cancer $[6,12]$ and also over the combination of an Al plus tamoxifen in the adjuvant setting [7], has indeed been confirmed in several key clinical trials. However, contrary to the preclinical model prediction, two recent randomized phase III trials comparing anastrozole with fulvestrant showed either that fulvestrant is as effective as anastrozole [13] or that fulvestrant is superior to anastrozole [14] for advanced breast cancer in postmenopausal women. Such discrepancies between the preclinical model and results from human trials may be related to some differences in doses, artifacts of single-cell-line analysis, or intrinsic differences in pharmacokinetics and metabolism of drugs between human and mouse. These discrepancies emphasize some limitations of preclinical models in general, and of the intratumoral aromatase model in particular, and should be taken into consideration when translating these findings into the clinic.

\section{Sequential therapy after first-line treatment with aromatase inhibitor}

The establishment of the optimal sequence of endocrine therapies offers significant benefits to women with hormone-sensitive metastatic breast cancer by prolonging the treatment period during which HT can be used [8]. In a recent study, Long and colleagues, using their intratumoral aromatase model, investigated the optimal second-line therapy for patients who fail Al therapy [2]. The authors have taken both in vitro and in vivo approaches to define hormonal sensitivities of these Al-resistant tumor cells. In vitro, aromatase-transfected MCF-7Ca cells were selected by long-term estrogen deprivation to grow in estrogendepleted medium (UMB-1Ca). Aromatase activity in these cells did not significantly change. Whole-cell ligand-binding assays showed that these selected cells expressed elevated levels of functionally active ER. Basal ER-transcriptional activity was not affected in these cells (as assessed by the level of progesterone receptor), but estrogen stimulation led to a much higher induction of ER activity than in parental cells. Importantly, however, this increased ER activity did not translate to an increased growth response to estrogen, and the cells did not show hypersensitivity to low estrogen levels, contrasting what was previously shown with estrogen-deprived hormone-resistant MCF-7 cells that do not overexpress aromatase [15]. Nevertheless, like the parental MCF-7 cells [15], UMB-1Ca cells remained sensitive to anti-estrogens, both in vitro and in vivo.

To model the clinical situation of treating postmenopausal breast cancer patients after the failure of $\mathrm{Al}$, Long and colleagues took a second approach and defined the anti-estrogen sensitivity of letrozole-resistant MCF-7Ca xenografts. Resistant xenografts were first selected by their acquired ability to grow in the presence of the letrozole after long-term treatment; the mechanism of resistance has yet to be defined. Resistant tumors were transplanted and reestablished in naive nude mice in the presence of aromatase substrate, and mice were then treated with either anti-estrogens or Als. Transplant growth was slowed significantly by tamoxifen and even more effectively by the potent anti-estrogen fulvestrant. Surprisingly, while these resistant tumors were refractory to the Als anastrozole and formestane, they regained significant sensitivity to letrozole. The results therefore suggest that tumors that have failed Al therapy would remain sensitive to second-line therapy with anti-estrogens, and that they may also 
respond to rechallenge with $\mathrm{Al}$ therapy. Some clinical data have also suggested the possibility that resistance to nonsteroidal Als would not confer cross-resistance to the steroidal Als, and vice versa [8]. Whether the increased cellular ER levels in the resistant tumors are responsible for their enhanced sensitivity to anti-estrogen is still an open question. Without doubt, further studies to investigate the underlying mechanisms responsible for letrozole resistance are crucial for a better understanding of how to circumvent or overcome this resistance in the clinic.

\section{The SERM arzoxifene in breast cancer prevention and treatment}

Since estrogens have a crucial role in breast cancer initiation, proliferation, and metastasis [16], Als and parallel methods to eliminate endogenous estrogen may offer a potent strategy for breast cancer treatment and prevention. However, estrogens also play an indispensable role in other organs [17], and therefore SERMs, which possess anti-estrogen activity in the breast but preserve some fundamental estrogen-like functions in other tissues, may offer a more comprehensive and balanced tactic. While tamoxifen was the first widely used breast cancer prevention agent, and is still, along with raloxifene, under active clinical testing for prevention applications, novel SERMs with a more desirable tissue profile are needed. Because of significant advances in understanding the structure and function of ER, several leading and promising compounds are now under clinical development.

As discussed in two recent reports by Suh and colleagues of Sporn's group [3,18], the SERM arzoxifene (Arz) is one of the more promising compounds. It is a new benzothiophene derivative similar to raloxifene. Arz acts as a potent anti-estrogen in breast and uterine tissues [19] but possesses estrogen agonist activity to maintain bone density and serum cholesterol. In comparison with tamoxifen, Arz has shown a stronger and more durable antagonist activity in breast cancer cell lines and xenografts [20,21]. Several recent phase II clinical trials also reported high antitumor activity of Arz in advanced breast and endometrial cancers [22]. In their first report regarding Arz, Suh and colleagues showed that Arz is a highly effective agent that is significantly more potent than raloxifene for the prevention of mammary tumors in a rat model [18]. All of the above studies suggest that the SERM profile of Arz is excellent, and that this agent therefore has great promise for breast cancer treatment and prevention.

\section{Arzoxifene in combination with the rexinoid LG 100268 (LG268)}

Recent preclinical studies have revealed the potential of combinations of HT with other agents for both prevention and treatment of breast cancer. These agents may act indirectly through the ER, mainly by influencing ER bidirec- tional crosstalk with other signaling pathways, or may act independently of the ER pathway and affect stromal as well as epithelial cells [23]. The rexinoids, selective ligands for the retinoid $X$ receptors [24], are more effective in tissues that contain ER protein [25] and they exhibit great potential for both chemoprevention and therapy of cancer. Recent studies have also shown that rexinoids can prevent ER-negative breast cancer as well [26].

In a follow-up report on Arz, Suh and colleagues [3] now demonstrate the high efficacy as single agents, and the striking synergy when combined, of Arz and the new rexinoid compound LG268 [24] for both treatment and prevention of breast cancer. The authors again used the $\mathrm{N}$-nitrosomethylurea (NMU) rat model, an established model for breast cancer development and progression that produces hormone-dependent tumors [27] and has been used successfully to predict the efficacy of chemopreventive hormonal agents. Prevention efficacy against either early or more advanced premalignant lesions was tested by starting the treatment either early or late after the administration of NMU. Prevention of progression of early lesions, which was first demonstrated for the LG268 compound in that paper, was dosage dependent for both drugs. Importantly, when the two drugs were combined, only very low dosages of both were needed to cause meaningful reduction in tumor burden, a significant observation for the clinical setting to minimize toxicity. The high efficacy of each of the drugs and their synergism, though with higher dosages than in the prevention setting, was further achieved in the treatment setting for both early and advanced invasive breast cancer. As a single agent, each drug was capable of slowing or arresting tumor growth, but when the drugs were combined, a dramatic synergism was seen, which resulted in almost complete tumor shrinkage of even very large tumors in as short a time as 3 weeks after the beginning of treatment.

Although some of the inhibitory effects of both Arz and LG268 are very likely due to direct actions on premalignant or malignant mammary epithelial cells, Suh and colleagues hypothesized that part of the synergistic effect may also be mediated by an effect on the stromal cells that control the microenvironment of the tumor. To investigate this hypothesis, they conducted several experiments. Fibroblast cells were treated with Arz and LG268, either alone or in combination, and the effect of their conditioned medium on the growth of lung epithelial cells highly sensitive to transforming growth factor $\beta$ (TGF- $\beta$ ) [28] was monitored. Growth inhibition of the epithelial cells was induced only by medium derived from fibroblasts treated with the combination of Arz and LG268, and not when the drugs were administered as single agents. This inhibitory effect was most likely related to TGF- $\beta$, since a blocking antibody to TGF- $\beta$ completely reversed the inhibition. Furthermore, by examining induction of the important 
angiogenic factor inducible nitric oxide synthase (iNOS) in the fibroblast cells, which is known to be inhibited by TGF- $\beta$ [29], the authors found that the induction was almost entirely blocked by the combination of Arz and LG268, whereas the compounds had little effect on the expression of iNOS when used alone. Obviously, other mechanistic studies, especially in the context of the NMUrat model, are needed to investigate further the intriguing hypothesis that stromal TGF- $\beta$ induction [30] is the conduit by which Arz and LG268 synergistically induce tumor regression, and to reveal other underlying mechanisms that are responsible for this synergistic action on breast cancer prevention and treatment. But regardless of these future studies, the data discussed above, as also concluded by the researchers, strongly suggest that clinical application of the combination of Arz and LG268 should be considered for prevention as well as for treatment in both the adjuvant and advanced breast cancer settings.

\section{Competing interests}

None declared.

\section{Acknowledgements}

This work was supported in part by a SPORE grant (NCI SPORE P50 CA 50183).

\section{References}

1. O'Regan RM, Jordan VC: The evolution of tamoxifen therapy in breast cancer: selective oestrogen-receptor modulators and downregulators. Lancet Oncol 2002, 3:207-214.

2. Long BJ, Jelovac D, Thiantanawat A, Brodie AM: The effect of second-line antiestrogen therapy on breast tumor growth after first-line treatment with the aromatase inhibitor letrozole: long-term studies using the intratumoral aromatase postmenopausal breast cancer model. Clin Cancer Res 2002, 8:2378-2388.

3. Suh N, Lamph WW, Glasebrook AL, Grese TA, Palkowitz AD, Williams CR, Risingsong R, Farris MR, Heyman RA, Sporn MB: Prevention and treatment of experimental breast cancer with the combination of a new selective estrogen receptor modulator, arzoxifene, and a new rexinoid, LG 100268. Clin Cancer Res 2002, 8:3270-3275.

4. Emmens CW, Cox Rl, Martin L: Antiestrogens. Recent Prog Horm Res 1962, 18:415-466.

5. Brodie A, Long B: Aromatase inhibition and inactivation. Clin Cancer Res 2001, 7:4343s-4349s; discussion 4411s-4412s.

6. Goss P: Anti-aromatase agents in the treatment and prevention of breast cancer. Cancer Control 2002, 9:2-8.

7. Baum M, Budzar AU, Cuzick J, Forbes J, Houghton JH, Klijn JG, Sahmoud T, ATAC Trialists' Group: Anastrozole alone or in combination with tamoxifen versus tamoxifen alone for adjuvant treatment of postmenopausal women with early breast cancer: first results of the ATAC randomised trial. Lancet 2002, 359:2131-2139.

8. Carlson RW: Sequencing of endocrine therapies in breast cancer - integration of recent data. Breast Cancer Res Treat 2002, 75:S27-S32; discussion S33-S25.

9. Miller WR, Hawkins RA, Forrest AP: Significance of aromatase activity in human breast cancer. Cancer Res 1982, 42:3365s3368s.

10. Yue W, Wang J, Savinov A, Brodie A: Effect of aromatase inhibitors on growth of mammary tumors in a nude mouse model. Cancer Res 1995, 55:3073-3077.

11. Brodie A, Jelovac D, Long BJ: Predictions from a preclinical model: studies of aromatase inhibitors and antiestrogens. Clin Cancer Res 2003, 9:455S-459S.

12. Buzdar AU: Anastrozole (Arimidex) in clinical practice versus the old 'gold standard', tamoxifen. Expert Rev Anticancer Ther 2002, 2:623-629.
13. Howell A, Robertson JF, Quaresma Albano J, Aschermannova A, Mauriac L, Kleeberg UR, Vergote I, Erikstein B, Webster A, Morris C: Fulvestrant, formerly $\mathrm{ICl} 182,780$, is as effective as anastrozole in postmenopausal women with advanced breast cancer progressing after prior endocrine treatment. J Clin Oncol 2002, 20:3396-3403.

14. Osborne CK, Pippen J, Jones SE, Parker LM, Ellis M, Come S, Gertler SZ, May JT, Burton G, Dimery I, Webster A, Morris C, Elledge R, Buzdar A: Double-blind, randomized trial comparing the efficacy and tolerability of fulvestrant versus anastrozole in postmenopausal women with advanced breast cancer progressing on prior endocrine therapy: results of a North American trial. J Clin Oncol 2002, 20:3386-3395.

15. Masamura S, Santner SJ, Heitjan DF, Santen RJ: Estrogen deprivation causes estradiol hypersensitivity in human breast cancer cells. J Clin Endocrinol Metab 1995, 80:2918-2925.

16. Clemons M, Goss P: Estrogen and the risk of breast cancer. $N$ Engl J Med 2001, 344:276-285.

17. Grady D, Rubin SM, Petitti DB, Fox CS, Black D, Ettinger B, Ernster VL, Cummings SR: Hormone therapy to prevent disease and prolong life in postmenopausal women. Ann Intern Med 1992, 117:1016-1037.

18. Suh N, Glasebrook AL, Palkowitz AD, Bryant HU, Burris LL, Starling JJ, Pearce HL, Williams C, Peer C, Wang Y, Sporn MB: Arzoxifene, a new selective estrogen receptor modulator for chemoprevention of experimental breast cancer. Cancer Res 2001, 61:8412-8415.

19. Vernon MW: Experimental endometriosis in laboratory animals as a research model. Prog Clin Biol Res 1990, 323:49-60.

20. Schafer JM, Lee ES, Dardes RC, Bentrem D, O'Regan RM, De Los Reyes A, Jordan VC: Analysis of cross-resistance of the selective estrogen receptor modulators arzoxifene (LY353381) and LY117018 in tamoxifen-stimulated breast cancer xenografts. Clin Cancer Res 2001, 7:2505-2512.

21. Chan S: Arzoxifene in breast cancer. Eur J Cancer 2002, 38: S55-S56.

22. Buzdar A, O'Shaughnessy JA, Booser DJ, Pippen JE Jr, Jones SE, Munster PN, Peterson P, Melemed AS, Winer E, Hudis C: Phase II, randomized, double-blind study of two dose levels of arzoxifene in patients with locally advanced or metastatic breast cancer. J Clin Oncol 2003, 21:1007-1014.

23. Fabian CJ, Kimler BF: Chemoprevention for high-risk women: tamoxifen and beyond. Breast $J$ 2001, 7:311-320.

24. Boehm MF, Zhang L, Zhi L, McClurg MR, Berger E, Wagoner M, Mais DE, Suto CM, Davies JA, Heyman RA, Nadzan AM: Design and synthesis of potent retinoid $X$ receptor selective ligands that induce apoptosis in leukemia cells. J Med Chem 1995, 38:3146-3155.

25. van der Leede BJ, Folkers GE, van den Brink CE, van der Saag PT, van der Burg B: Retinoic acid receptor alpha 1 isoform is induced by estradiol and confers retinoic acid sensitivity in human breast cancer cells. Mol Cell Endocrinol 1995, 109:77-86.

26. Wu K, Zhang Y, Xu XC, Hill J, Celestino J, Kim HT, Mohsin SK, Hilsenbeck SG, Lamph WW, Bissonette R, Brown PH: The retinoid $X$ receptor-selective retinoid, LGD1069, prevents the development of estrogen receptor-negative mammary tumors in transgenic mice. Cancer Res 2002, 62:6376-6380.

27. Russo J, Gusterson BA, Rogers AE, Russo IH, Wellings SR, van Zwieten MJ: Comparative study of human and rat mammary tumorigenesis. Lab Invest 1990, 62:244-278.

28. Danielpour D, Dart LL, Flanders KC, Roberts AB, Sporn MB: Immunodetection and quantitation of the two forms of transforming growth factor-beta (TGF-beta 1 and TGF-beta 2) secreted by cells in culture. J Cell Physiol 1989, 138:79-86.

29. Vodovotz Y, Bogdan C, Paik J, Xie OW, Nathan C: Mechanisms of suppression of macrophage nitric oxide release by transforming growth factor beta. J Exp Med 1993, 178:605-613.

30. Derynck R, Akhurst RJ, Balmain A: TGF-beta signaling in tumor suppression and cancer progression. Nat Genet 2001, 29:117129.

\section{Correspondence}

Rachel Schiff, PhD, Breast Center - room N1230.01, Baylor College of Medicine, One Baylor Plaza (BCM 600), Houston TX 77030, USA. Tel: +1 713798 1676; fax: +1 713798 1673; e-mail: rschiff@breastcenter.tmc.edu 